\title{
Capacidad cognitiva y percepción de calidad de vida de personas entre 54 y 78 años de edad que participaron en actividades recreativas
}

\section{Cognitive ability and perceived quality of life for people between 54 and 78 years who participated in recreational activities}

\author{
Sigrid Vargas Méndez \\ Bachiller en Educación Física \\ Universidad de Costa Rica \\ Costa Rica \\ sigridvargasmendez@gmail.com
}

Recibido: 02-XI-2011 • Aceptado 17-XI-2011 • Corregido 25-VI-2011

\begin{abstract}
Resumen: El objetivo de este estudio fue evaluar la capacidad cognitiva y la percepción de calidad de vida de las personas adultas mayores que participaron en un grupo de baile folclórico de representación, uno de baile popular y en otro de actividades físicorecreativas, para determinar si el tipo de actividad recreativa en la que se participa influyó en estas dos variables. Se utilizó el cuestionario SF-36 de percepción de calidad de vida y el test de Raven. El estudio se realizó con una muestra de 39 mujeres, con edades comprendidas entre los 54 y 78 años. El ANOVA de 1 vía y el análisis post hoc de Tukey, aplicados a los datos obtenidos con el SF-36 y el Test de Raven, mostraron que la única diferencia significativa entre grupos se encontró en el componente de Salud General, entre el grupo de actividades físico-recreativas y el grupo de baile folclórico. El grupo de actividades recreativas mostró una percepción de su estado de Salud General más positiva que el grupo de bailes folclóricos. No hubo ninguna diferencia significativa con respecto a la capacidad cognitiva en los diferentes grupos de la investigación. Teniendo en cuenta el valor máximo que se podía obtener en esta prueba, los promedios estuvieron dentro del rango normal (entre o y 1 DS). Se concluyó que los tres grupos en estudio
\end{abstract}

\section{Introducción}

Según el foro Mundial de la Salud, realizado en Ginebra en 1996, la calidad de vida se define como la percepción del individuo sobre su posición en la vida dentro del contexto cultural y el sistema de valores en el que vive y con respecto a sus metas, expectativas, normas y preocupaciones. Es un concepto extenso y complejo que engloba la salud física, el estado psicológico, el nivel de independencia, las relaciones sociales, las creencias personales y la relación con las características sobresalientes del entorno (Grupo WHOQOL, 1996, p. 28).

Esta definición pone de manifiesto algunos criterios, los cuales afirman que la calidad de vida se refiere a una evaluación subjetiva, con dimensiones tanto positivas 
tienen una percepción de calidad de vida positiva y una capacidad cognitiva dentro del rango normal independientemente del tipo de actividad recreativa en que participaron. Los promedios obtenidos del Cuestionario de calidad de vida SF-36 en los tres grupos evaluados quedaron arriba del promedio de los valores de referencia españoles. Se concluye que se deben ofrecer programas recreativos que ofrezcan excelencia y eficacia hacia este grupo etario, tomando en cuenta las recomendaciones específicas sobre el tipo de actividades recreativas expuestas en este estudio.

Palabras clave: Envejecimiento, cognición, recreación, baile, calidad de vida.

\begin{abstract}
The goal of this research was to evaluate cognitive ability and the quality of life's perception of older persons who participated in a group of folkloric dance, one of Latin popular dance and other of physical and recreational activities, to determine if the type of recreational activity in which they participated influenced is these two variables. The SF-36 questionnaire perception of the quality of life and Raven test were used. The study was conduct with a sample of 39 women, aged from 54 to 78 years. The 1-way ANOVA and Tukey's post hoc analysis, applied to the data obtained with the SF-36 and Raven's Test showed that the only significant difference between groups was in the General Health component, between the group of physical and recreational activities and folkloric dance group. Recreational activities group showed more positive perception of general health status than the group of folk dances. No significant difference exists regarding the cognitive ability of the different research groups. Taking into account the maximum value that could be obtained in this test, the averages were within the normal range (between 0 and $+1 S D)$. In conclusion the three study groups have a perception of positive quality of life and cognitive ability within the normal range regardless of the type of activity involved. The mean of the quality of life questionnaire SF-36 in the three groups were assessed above the average of the Spanish reference values. It should offer recreational programs that provide excellence and efficiency to this age group, taking into account the specific recommendations on the type of recreational activities outlined in this study.
\end{abstract}

Key words: Aging, cognition, recreation, dance, quality of life. como negativas, y que está arraigada a un contexto cultural, social y ambiental. La Organización Mundial de la Salud (OMS, citada por Nutbeam, 1997) ha identificado seis extensas áreas que describen aspectos fundamentales de la calidad de vida en todas las culturas: un área física, un área psicológica, el nivel de independencia, las relaciones sociales, el entorno y las creencias personales/espirituales.

La calidad de vida refleja la percepción que tienen los individuos de que sus necesidades están siendo satisfechas, o bien de que se les están negando oportunidades para alcanzar la felicidad y la autorrealización, con independencia de su estado de salud o de las condiciones sociales y económicas (Nutbeam, 1997).

La definición de la Organización Mundial de la salud (OMS) utiliza un concepto de salud (estado de completo bienestar físico, mental y social, y no solamente la ausencia de afecciones o enfermedades) que incluye tanto la salud física como la mental, relacionando ambos conceptos (OMS, 1986). De esta forma, la mayoría de los autores proponen que el envejecimiento saludable se debe definir con base en el buen funcionamiento físico y mental, la capacidad para llevar una vida independiente, una buena función cognitiva y la ausencia de depresión (Puga, Rosero, Glaser, y Castro, 2007).

Existen muchas teorías que buscan explicar cuándo empieza y en qué consiste el proceso de envejecimiento, teorías históricas, orgánicas y genéticas. Cada una da su aporte para comprender mejor el proceso de envejecimiento, el cual es un proceso fisiológico asociado a un proceso natural común a todos los seres vivos (Fernández García, 2005). La ley integral para la persona adulta mayor ( $\mathrm{N}^{\circ}$ 7935) define como persona adulta mayor a toda persona mayor de sesenta y cinco años o más; sin embargo, en ocasiones personas menores (menores a 65 años) participan en programas creados para adultos mayores por el hecho de estar jubiladas, retiradas 
del ambiente laboral o por tener alguna limitación física que afecte su movilidad o sus hábitos diarios en general.

Las recomendaciones del Colegio Colegio Americano de Medicina Deportiva (ACSM) y la Asociación Americana del Corazón (AHA) aplican a personas de 65 años o mayores, así como para adultos con edades de 50-64 años de edad con condiciones crónicas clínicamente significativas o limitaciones funcionales que afecten la habilidad de movimiento, aptitud física, o actividad física (ACSM, 2007).

Debido a que la senectud no evoluciona de la misma manera en toda la población, al examinar los diversos procesos del envejecimiento, se debe tomar en cuenta edades menores que las establecidas por la ley (65 años) y así considerar también a las personas afectadas por condiciones crónicas clínicamente significativas (ACSM, 2007).

Los cambios o alteraciones fisiológicas que ocurren en el cuerpo al envejecer están adscritos genéticamente a modificaciones en los sistemas claves del organismo: cardíaco, nervioso y endocrino. Respecto al declive del sistema nervioso central en la vejez, no se deteriora la capacidad organizativa ni el contenido de los procesos mentales como producto del envejecimiento normal, pero sí se presentan modificaciones que influirán en el funcionamiento normal de los procesos cognitivos (Carazo Vargas, 2004).

Para que el cerebro funcione de manera óptima y eficiente, no solo hacen falta células nerviosas y neurotransmisores en buen estado, sino que también es necesario que esté bien irrigado por la sangre, que utilice bien el oxígeno y la glucosa y que disponga de las enzimas necesarias para las reacciones bioquímicas que van a permitir la transmisión de la información de una neurona a otra (Pont Geis, 2007).

El ejercicio es un mecanismo ideal y necesario para el funcionamiento óptimo del cuerpo. Algunos mecanismos biológicos que explican los beneficios del ejercicio son el aumento del suministro de oxígeno a nivel cerebrovascular, incremento del flujo sanguíneo en todo el cuerpo y a nivel coronario, disminución de la frecuencia cadíaca y la presión arterial, aumento de la estabilidad eléctrica del corazón al disminuir la isquemia e incremento del umbral de fibrilación (arritmias). Además de lo anterior, el ejercicio mejora la tolerancia a la isquemia, incrementa la capacidad antioxidante del miocardio (remoción de radicales libres) en pacientes con diabetes, aumenta la sensibilidad a la insulina y contribuye en el control de la glicemia (Wilmore y Costill, 2001).

Pont Geis (2007) enumera seis alteraciones que se producen de manera progresiva con el paso de la edad y que influyen directamente con lo mencionado anteriormente:

\footnotetext{
La cantidad de sangre que irriga el cerebro disminuye.

El consumo de glucosa y de oxígeno por las neuronas se reduce en un $10 \%$.

En las neuronas aparecen corpúsculos de lipofusina, o bien "pigmentos de selenidad".

El número de neuronas disminuye de un 25 a un $45 \%$ en la corteza cerebral.

Aparecen modificaciones estructurales en las neuronas y sus conexiones.

Los neurotransmisores disminuyen.
}

Múltiples estudios han demostrado que los programas de actividad física $\mathrm{y}$ recreación traen múltiples beneficios físicos y psicológicos a la persona adulta mayor y mejora su calidad de vida (Díaz, Díaz, Acuña, Donoso y Nowogrodsky, 2002; Martin, Powell, Peel, Zhu y Allman, 2006; Gracia y Marcó, 2000). En un programa piloto de actividad física integral desarrollado en Chile, se reportó que en promedio, las personas obesas disminuyeron el Índice de Masa Corporal (IMC) en 1,14; las hipertensas, la presión arterial sistólica en 4,38 $\mathrm{mmHg}$ y la presión arterial diastólica en 2,99 $\mathrm{mmHg}$; y las diabéticas, la glicemia en $6,75 \mathrm{mg} / \mathrm{dl}$. Al término del programa, un $95,3 \%$ de las personas participantes se sintieron más ágiles y un $93,1 \%$ con mejor salud (Salinas, Bello, Flores, Carbullanca y Torres, 2005). Los programas efectivos de ejercicio y/o actividad física para personas 
adultas mayores deben orientarse a combatir la fragilidad y vulnerabilidad causada por la inactividad, a minimizar los cambios biológicos del envejecimiento, controlar enfermedades crónicas, maximizar la salud emocional, incrementar la movilidad y otros. Idealmente, la prescripción de ejercicio para adultos mayores debe incluir ejercicio aeróbico, ejercicios de resistencia (fuerza) y ejercicios de flexibilidad (ACSM, 2007).

La búsqueda constante de salud y bienestar del ser humano debe ir encaminada a la mejoría de la calidad de vida, en la cual la recreación es una opción esencial.

El papel de la recreación y el buen manejo del tiempo libre son fundamentales para la consecución de un alto nivel de satisfacción con la vida, lo que equivale a un éxito en la adaptación a la jubilación (Nimrod, 2007).

La recreación, como menciona Salazar Salas (2007), es un conjunto de actividades agradables que producen gozo, realizadas durante el tiempo libre, que además puede ayudar a prevenir enfermedades como derrame cerebral, el cáncer de colon y diabetes, entre otros problemas de salud. Puede ser practicada en familia o de forma individual.

Según Rossman y Schlatter (citados por Salazar Salas, 2007), la recreación tiene un propósito moral en la sociedad y una restauración del arduo trabajo.

La actividad física en la persona adulta mayor puede ser considerada también como actividad recreativa que este grupo etario podría realizar con el fin de lograr otros objetivos (no fisiológicos), como por ejemplo: liberación de tensiones emocionales, reafirmación personal e interacción social (Díaz, Díaz, Acuña, Donoso y Nowogrodsky, 2002). Así mismo, el baile es clasificado como actividad recreativa por sus características. Ballestero (1998) cita que la recreación debe contar con al menos cinco características: 1 . darse en el tiempo libre; 2. ser voluntaria; 3. producir satisfacción 4. ser constructiva y 5. aceptada por la sociedad.
Así, tanto la actividad física como el baile contribuyen a una mejor calidad de vida en la persona adulta mayor, donde la salud y la capacidad funcional son de vital importancia para un envejecimiento digno. El nivel de capacidad funcional determina la medida en que pueden manejarse con autonomía dentro de la comunidad, participar en distintos eventos, visitar a otras personas, utilizar los servicios y en general, enriquecer sus propias vidas y las de las personas más próximas a ellos (OMS, 1998).

Por lo tanto, se debe dotar a este colectivo de diversidad y calidad en los programas que se les ofrecen, evaluando el impacto de la participación sobre su competencia funcional e investigando constantemente posibles innovaciones, buscando siempre la excelencia y efectividad de los mismos.

\section{Objetivos}

\section{Objetivo general}

El objetivo de este estudio fue evaluar la percepción de la calidad de vida y la capacidad cognitiva de las personas adultas mayores que participaron en uno de los siguientes tres grupos:

\section{Baile folklórico de representación \\ Baile popular \\ Actividades físico-recreativas}

\section{Objetivo específico}

Analizar las dimensiones que compone el concepto de calidad de vida y la capacidad cognitiva en los diferentes grupos de la investigación para determinar si existen diferencias en la percepción de la calidad de vida entre los grupos mencionados anteriormente.

\section{Metodología}

\section{Tipo de investigación}

El diseño de la investigación fue post facto, ya que no se aplicó ningún tratamiento a los sujetos. 


\section{Muestra}

Participaron 39 mujeres, con edades comprendidas entre 54 y 78 años, con un promedio de edad de 65,4 años. Cada una de ellas pertenece a uno de los siguientes grupos:

Grupo de Baile folklórico: ensayan tres horas a la semana, un mismo día, con receso y merienda a la mitad del ensayo. Realizan montajes coreográficos para presentarse en actividades especiales. Catorce mujeres participaron en el estudio.

Grupo de baile popular: reciben una clase intensiva de varios géneros musicales, durante una hora, una vez por semana. Algunas habían participado antes en algún grupo similar, para otras es una nueva experiencia. El grupo se formó hace 5 meses aproximadamente. Diez mujeres participaron en la investigación.

Grupo de actividades físico-recreativas: con dos clases a la semana de 1 hora y 50 minutos cada una. Realizan juegos, dinámicas, ejercicios, cantos y actividades variadas, principalmente en grupo. La mayoría habían llevado el mismo curso el semestre anterior. El grupo estaba conformado por 25 personas, sin embargo se tomó en cuenta sólo a la población femenina que asistió al día de la evaluación (15 mujeres).

\section{Instrumentos}

En esta investigación se utilizaron dos instrumentos:

1) Cuestionario de salud SF-36

2) Test de Raven

1) SF-36: Cuestionario muy utilizado para evaluar la calidad de vida, debido a su simplicidad y corto tiempo de aplicación. Contiene 36 ítems divididos en ocho dimensiones:

Función física: 3, 4, 5, 6, 7, 8, 9, 10, 11, 12 .

Vitalidad: 23, 27, 29 y 31.

Desempeño físico: 13, 14, 15, 16.

Dolor físico: 21 y 22.

Salud general: 1, 33, 34, 35,36.

Función social: 20 y 32

Desempeño emocional: 17, 18, 19.

Salud mental: 24, 25, 26, 28 y 30 .
Para esta investigación se aplicó el cuestionario de Salud SF-36 (versión española, modificada por Madrigal Jiménez, 2010), para evaluar las ocho dimensiones que el instrumento contempla.

Cada pregunta recibe un valor que posteriormente se transforma en una escala de 0 a 100, donde 0 corresponde al peor valor de calidad de vida y 100 a la salud perfecta.

La fórmula para realizar la transformación es la siguiente:

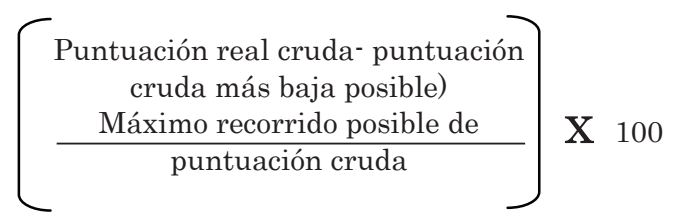

Evaluación del ítem de la Salud General: Las respuestas "Muy Buena" y "Buena" del ítem se recalibran (unen) para conseguir un mejor ajuste final con el concepto de evaluación de la salud general de esta escala. Según Medical Outcomes Trust (2000), de los estudios realizados hasta la publicación de este manual de puntuación, de los valores medios para la escala de criterio general de la salud para encuestados que eligen cada uno de los cinco niveles definidos por el ítem 1 se desvían significativamente de la linealidad. Los estudios empíricos en relación con el Estudio de los Seguros Médicos de Rand Health Insurance Experiment (HIE) fueron de los primeros en documentar que los intervalos entre opciones de respuesta a este ítem no son iguales (Davies y Ware, 1981; citados en Medical Outcomes Trust, 2000).

2) Test de Raven: versión corta (de 15 placas), la persona utiliza habilidades perceptivas, de observación y de razonamiento analógico para deducir el faltante en la matriz. Se le pide a la persona que analice la serie que se le presenta y que, siguiendo la secuencia horizontal y vertical, escoja uno de los ocho trazos; el que encaje perfectamente en ambos sentidos, tanto en el horizontal como en el vertical. Se evalúan las matrices elegidas comparándolas con las respuestas correctas. El Raven mide la capacidad 
intelectual para comparar formas y razonar por analogías, independientemente de los conocimientos adquiridos. De esta manera brinda información sobre la capacidad y claridad de pensamiento presente de la persona en estudio para la actividad intelectual.

\section{Recolección de datos}

Antes de comenzar la actividad recreativa (actividad física, baile popular o baile folclórico) se les aplicó el cuestionario SF-36, se les explicó en qué consistía y se les brindó el tiempo necesario para contestarlo. La siguiente semana se aplicó el Test de Raven. Al ser esta una prueba con un nivel de complejidad de mediano a alto, se brindó un ejemplo con dos de las matrices que no se encontraban dentro de la versión corta.

\section{Análisis de datos}

Los resultados de cada uno de los instrumentos aplicados se transcribieron a una hoja de cálculo. Luego de la recolección de datos, se realizó un análisis de varianza de una vía para grupos independientes y se comparó cada una de las ocho dimensiones del SF- 36 y el puntaje total obtenido del test de Raven; entre los tres grupos de sujetos, se tomó en cuenta dentro del análisis de varianza el factor edad para poder determinar si este influyó sobre los resultados de las otras dos variables (SF-36 y Raven).

En cuanto al Test de Raven, se obtuvo los valores promedio logrados por cada grupo, se convirtió la cantidad de puntos correctos de las 15 matrices a su equivalente porcentual (el puntaje máximo era $15 \mathrm{y}$ éste equivale al 100\%) y se convirtieron los valores promedio de cada grupo en porcentajes. Asimismo, se calculó los puntajes Z de los promedios obtenidos en cada grupo con respecto a la media del puntaje total de la prueba $(15 / 2=7,5)$ y la Desviación Estándar (DS) se calculó aplicando regla de tres (área entre 0 y una $\mathrm{DS}=4,13 \%$ ).

\section{Hipótesis}

Existe diferencia significativa en cuanto a percepción de calidad de vida y a capacidad cognitiva entre los tres grupos de personas: grupo de baile popular, grupo de baile folclórico de representación y grupo de actividades físico-recreativas.

En la Tabla 1 se presentan los valores de cada dimensión para realizar la conversión y sustituir los valores en la fórmula mencionada anteriormente.

\section{Resultados}

A continuación se presenta los resultados obtenidos del análisis de datos de cada uno de los instrumentos utilizados; en la tabla 2, 3 y 4 se presenta un análisis descriptivo de promedios y desviaciones estándar de la edad, cuestionarios SF-36 y test de Raven, respectivamente. En la tabla 5 se presentan los resultados del análisis estadístico (ANOVA de 1 vía) donde se comparan los tres grupos en estudio.

En la tabla 2 se describen los promedios de edad de los tres grupos de estudio; por los resultados de este análisis, se planteó la incertidumbre de si la variable de edad podría influir directamente sobre la percepción de calidad de vida y sobre las habilidades perceptivas, de observación y de razonamiento analógico, debido a la gran diferencia entre mínimos y máximos de cada grupo; sin embargo, posteriormente y mediante el análisis de varianza se eliminó la incertidumbre.

En la tabla 4 se indican las puntuaciones alcanzadas para cada una de las dimensiones del cuestionario SF-36. Los promedios variaron entre grupos y en todas las dimensiones excepto en la Función social, el grupo de baile folclórico obtuvo los promedios más bajos de los tres grupos de estudio. En el gráfico 2 se muestra el comportamiento de los promedios del cuestionario SF-36. 
Tabla 1

Valores de puntuaciones para realizar la transformación a la escala de 0 a 100 en el instrumento SF-36

\begin{tabular}{lccc}
\hline Escala & $\begin{array}{c}\text { Frecuencia Puntuaciones crudas } \\
\text { mínimas y máximas posibles }\end{array}$ & $\begin{array}{c}\text { Máximo recorrido posible de la } \\
\text { puntuación cruda }\end{array}$ \\
\hline Función física & 10 & Máx. & 20 \\
Desempeño físico & 4 & 30 & 4 \\
Dolor Corporal & 2 & 8 & 10 \\
Salud General & 5 & 25 & 20 \\
Vitalidad & 4 & 24 & 20 \\
Función Social & 2 & 10 & 3 \\
Desempeño emocional & 3 & 6 & 25 \\
Salud Mental & 5 & 30 & 3 \\
\hline
\end{tabular}

Fuente: Medical Outcomes Trust, 2000.

Tabla 2

Análisis descriptivo de la edad en los tres grupos de estudio

\begin{tabular}{llccccc}
\hline \multicolumn{1}{c}{ Grupo } & N. & Promedios & $\begin{array}{c}\text { Desviación } \\
\text { Std. }\end{array}$ & Mínimo & Máximo \\
\hline \multirow{2}{*}{ Edad (años) } & Baile popular & 10 & 65,4 & 3,78 & 58,00 & 71,00 \\
& $\begin{array}{l}\text { Actividades } \\
\text { recreativas }\end{array}$ & 15 & 64,2 & 5,68 & 54,00 & 78,00 \\
& Baile folclórico & 14 & 66,71 & 4,69 & 58,00 & 74,00 \\
& Total & 39 & 65,41 & 4,93 & 54,00 & 78,00 \\
\hline
\end{tabular}

Tabla 3

Puntuaciones del Test de Raven en los tres grupos de estudio

\begin{tabular}{lcc}
\hline $\begin{array}{l}\text { Desviación } \\
\text { Std }\end{array}$ & Mínimo & Máximo \\
\hline 1,95505 & 9 & 15 \\
2,66726 & 5 & 15 \\
2,31099 & 6 & 14 \\
2,36740 & 5 & 15 \\
\hline
\end{tabular}


Gráfico 1

Porcentaje obtenido en TestRaven

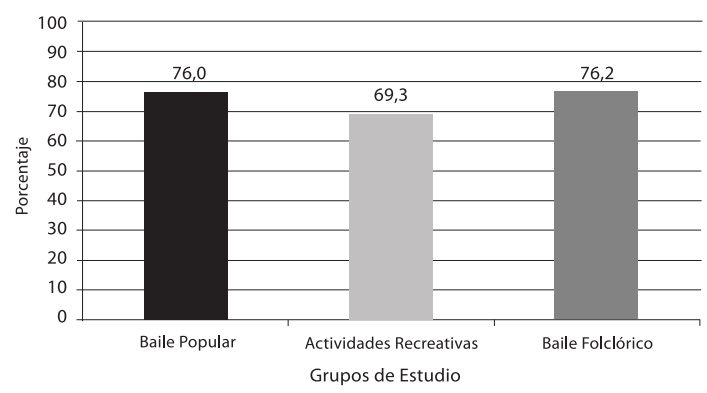

Tabla 4

Puntuaciones del Cuestionario SF-36 por dimensiones en los tres grupos de estudio

\begin{tabular}{|c|c|c|c|c|}
\hline & Grupo & $\mathrm{N}$. & Promedios & Desviación Std. \\
\hline \multirow{4}{*}{$\begin{array}{l}\text { Cambio en } \\
\text { percepción de salud } \\
\text { con respecto a un } \\
\text { año antes }\end{array}$} & Baile popular & 10 & 1,6 & 0,84327 \\
\hline & $\begin{array}{l}\text { Actividades } \\
\text { recreativas }\end{array}$ & 15 & 1,73 & 0,88372 \\
\hline & Baile folclórico & 14 & 2,35 & 1,00821 \\
\hline & Total & 39 & 1,92 & 0,95655 \\
\hline \multirow{4}{*}{ Función física } & Baile popular & 10 & 90,5 & 10,39498 \\
\hline & $\begin{array}{l}\text { Actividades } \\
\text { recreativas }\end{array}$ & 15 & 81 & 22,85045 \\
\hline & Baile folclórico & 14 & 78,57 & 13,92681 \\
\hline & Total & 39 & 82,56 & 17,54212 \\
\hline \multirow{4}{*}{ Función social } & Baile popular & 10 & 96,25 & 11,85854 \\
\hline & $\begin{array}{l}\text { Actividades } \\
\text { recreativas }\end{array}$ & 15 & 90 & 15,81139 \\
\hline & Baile folclórico & 14 & 90,18 & 15,64216 \\
\hline & Total & 39 & 91,66 & 14,71588 \\
\hline
\end{tabular}




\begin{tabular}{|c|c|c|c|c|}
\hline & Grupo & $\mathrm{N}$. & Promedios & Desviación Std. \\
\hline \multirow{4}{*}{ Vitalidad } & Baile popular & 10 & 84,5 & 11,16791 \\
\hline & $\begin{array}{l}\text { Actividades } \\
\text { recreativas }\end{array}$ & 15 & 81, & 16,92420 \\
\hline & Baile folclórico & 14 & 77,86 & 14,23893 \\
\hline & Total & 39 & 80,77 & 14,53392 \\
\hline \multirow{4}{*}{ Salud mental } & Baile popular & 10 & 90,8 & 13,73398 \\
\hline & $\begin{array}{l}\text { Actividades } \\
\text { recreativas }\end{array}$ & 15 & 87,73 & 16,17523 \\
\hline & Baile folclórico & 14 & 85,14 & 13,71612 \\
\hline & Total & 39 & 87,59 & 14,50357 \\
\hline \multirow{4}{*}{ Desempeño físico } & Baile popular & 10 & 100 & 0,00000 \\
\hline & $\begin{array}{l}\text { Actividades } \\
\text { recreativas }\end{array}$ & 15 & 88,33 & 24,76076 \\
\hline & Baile folclórico & 14 & 85,71 & 28,94671 \\
\hline & Total & 39 & 90,38 & 23,37860 \\
\hline \multirow{4}{*}{$\begin{array}{l}\text { Desempeño } \\
\text { emocional }\end{array}$} & Baile popular & 10 & 90 & 31,62278 \\
\hline & $\begin{array}{l}\text { Actividades } \\
\text { recreativas }\end{array}$ & 15 & 93,33 & 18,68706 \\
\hline & Baile folclórico & 14 & 92,86 & 26,72612 \\
\hline & Total & 39 & 92,31 & 24,73431 \\
\hline \multirow{4}{*}{ Dolor físico } & Baile popular & 10 & 88 & 13,88604 \\
\hline & $\begin{array}{l}\text { Actividades } \\
\text { recreativas }\end{array}$ & 15 & 79,63 & 24,42588 \\
\hline & Baile folclórico & 14 & 83,75 & 19,90724 \\
\hline & Total & 39 & 83,28 & 20,31003 \\
\hline \multirow{4}{*}{ Salud general } & Baile popular & 10 & 84,6 & 16,09141 \\
\hline & $\begin{array}{l}\text { Actividades } \\
\text { recreativas }\end{array}$ & 15 & 84,93 & 15,10188 \\
\hline & Baile folclórico & 14 & 71,64 & 12,83988 \\
\hline & Total & 39 & 80,07 & 15,57729 \\
\hline
\end{tabular}


Gráfico 2

Promedio de las dimesiones $>$ SF-36

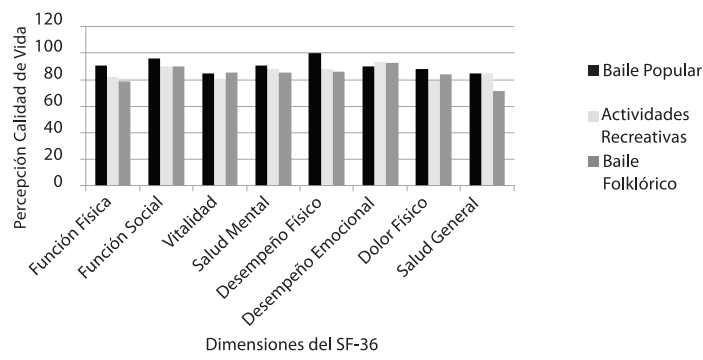

* Significativa con $\mathrm{p}<0,05$ entre actividades recreativas y baile folclórico

No en todas las dimensiones existió diferencia significativa, tan solo se evidenció en el componente de Salud General. En la tabla 5 se presentan los resultados por dimensión del Cuestionario de Salud SF-36 y el Test de Raven, además del análisis realizado a la edad de las participantes para demostrar que esta variable no tendría influencia en la diferencia entre grupos.

Tabla 5

Resultados de análisis estadístico (ANOVA de una vía, $\mathrm{N}=39$ ) en la comparación de los tres grupos de estudio para la edad. Test de Raven y Cuestionario SF-36

\begin{tabular}{lcc}
\hline \multicolumn{1}{c}{ Variable analizada } & F. & Sig. \\
\hline Edad en años & 0,950 & 0,396 \\
Cambio en percepción & 2,488 & 0,097 \\
de salud respecto al año & & \\
anterior & & \\
Función física & 1,482 & 0,241 \\
Función social & 0,640 & 0,533 \\
Vitalidad & 0,599 & 0,554 \\
Salud mental & 0,432 & 0,653 \\
Desempeño físico & 1,195 & 0,314 \\
Desempeño emocional & 0,057 & 0,945 \\
Dolor físico & 0,514 & 0,603 \\
Salud general & 3,649 & $0,036^{*}$ \\
Raven (puntos) & 0,845 & 0,438 \\
\hline
\end{tabular}

* Significativa con $p<0,05$
El ANOVA de una vía y el análisis post hoc de Tukey aplicados a los datos obtenidos con el SF-36 y el Test de Raven muestran que la única diferencia significativa entre grupos, existe en el componente de "salud general", entre el grupo de actividades recreativas y el grupo de baile folclórico. El grupo de actividades recreativas muestra una percepción más alta de su estado de salud general que el grupo de bailes folclóricos. Es importante aclarar que el factor edad no influyó en los resultados obtenidos del cuestionario de percepción de calidad de vida; a pesar de no establecer valores máximos y mínimos homogéneos en los grupos de estudio, no existe diferencia significativa entre la edad promedio de los grupos (Tabla 5). Por lo anterior, la edad no es una variable influyente ni explicativa en la percepción de calidad de vida de este estudio.

En lo que respecta a las demás dimensiones, los tres grupos de estudio mantuvieron promedios muy similares, por lo que no se encontraron diferencias significativas entre ninguna otra dimensión de calidad de vida. Cabe destacar que los valores promedios de cada dimensión se mantuvieron muy altos en los tres grupos de estudio y al comparar los obtenidos en esta investigación con otros estudios sobre el mismo cuestionario (Tabla 6), las mujeres de este estudio presentaron una percepción más positiva acerca de su calidad de vida.

Como se aprecia en el Gráfico 2, los promedios de los tres grupos estuvieron entre 69 y $76 \%$ del total de los puntos del Test de Raven. Al realizar este análisis se pudo determinar: con respecto a los puntos de la prueba que los promedios de los tres grupos se encontraban dentro de la zona normal (entre $0 \mathrm{y}+1 \mathrm{DS}$ ). Este dato indica que aunque no hay diferencia significativa entre los puntajes de los grupos, los tres tuvieron puntajes promedios normales con respecto a la escala de puntos del instrumento. 
Tabla 6

Comparación de valores promedio en la percepción de calidad de vida entre los resultados de esta investigación y los resultados en diferentes estudios que utilizaron el cuestionario SF-36

\begin{tabular}{|c|c|c|c|c|c|c|c|c|}
\hline Dimensión & $\begin{array}{l}\text { Función } \\
\text { física }\end{array}$ & $\begin{array}{c}\text { Función } \\
\text { social }\end{array}$ & Vitalidad & $\begin{array}{l}\text { Salud } \\
\text { mental }\end{array}$ & $\begin{array}{l}\text { Desempeño } \\
\text { físico }\end{array}$ & $\begin{array}{c}\text { Desempeño } \\
\text { emocional }\end{array}$ & $\begin{array}{l}\text { Dolor } \\
\text { físico }\end{array}$ & $\begin{array}{l}\text { Salud } \\
\text { general }\end{array}$ \\
\hline $\begin{array}{l}\text { Mujeres de } \\
\text { este estudio } \\
\text { Edad prom.: } \\
\mathbf{6 5 . 4} \text { años }\end{array}$ & 90,5 & 96,25 & 84,5 & 90,8 & 100 & 90 & 88 & 84,6 \\
\hline $\begin{array}{l}\text { Mujeres de } \\
\text { este estudio } \\
\text { Act. } \\
\text { Recreativas } \\
\text { Edad prom. } \\
\mathbf{6 4 . 2} \text { años }\end{array}$ & 81 & 90 & 81 & 87,733 & 88,333 & 93,333 & 79,6 & 84,933 \\
\hline $\begin{array}{l}\text { Mujeres de } \\
\text { este estudio } \\
\text { Baile } \\
\text { folclórico } \\
\text { Edad prom. } \\
\mathbf{6 6 . 7} \text { años }\end{array}$ & 78,5714 & 90,1786 & 77,8571 & 85,143 & 85,714 & 92,857 & 83,75 & 71,642 \\
\hline $\begin{array}{l}\text { México, } 1995 \\
\text { Mujeres } \\
55-64 \text { años, } \\
\text { usuarias } \\
\text { servicios } \\
\text { médicos }\end{array}$ & 93 & 100 & 89 & 40 & 50 & 67 & 100 & 53 \\
\hline México, 1995 & 89 & 84 & 77 & 72 & 87 & 86 & 81 & 85 \\
\hline $\begin{array}{l}\text { Mujeres } \\
55-64 \text { años, } \\
\text { institución } \\
\text { guberna- } \\
\text { mental }\end{array}$ & & & & & & & & \\
\hline $\begin{array}{l}\text { Referencia } \\
\text { versión } \\
\text { española } \\
\text { Edad: } 65-69 \\
\text { años }\end{array}$ & 68 & 79,2 & 58,7 & 64,8 & $\begin{array}{l}\text { Pendiente } \\
\text { de } \\
\text { desarrollo }\end{array}$ & $\begin{array}{l}\text { Pendiente } \\
\text { de } \\
\text { desarrollo }\end{array}$ & 66,1 & 54,7 \\
\hline $\begin{array}{l}\text { Referencia } \\
\text { versión } \\
\text { española } \\
\text { Edad: 70-74 } \\
\text { años }\end{array}$ & 63,1 & 76,5 & 56,3 & 62,7 & $\begin{array}{l}\text { Pendiente } \\
\text { de } \\
\text { desarrollo }\end{array}$ & $\begin{array}{l}\text { Pendiente } \\
\text { de } \\
\text { desarrollo }\end{array}$ & 61 & 52,7 \\
\hline
\end{tabular}




\begin{tabular}{|c|c|c|c|c|c|c|c|c|}
\hline Dimensión & $\begin{array}{c}\text { Función } \\
\text { física }\end{array}$ & $\begin{array}{c}\text { Función } \\
\text { social }\end{array}$ & Vitalidad & $\begin{array}{l}\text { Salud } \\
\text { mental }\end{array}$ & $\begin{array}{l}\text { Desempeño } \\
\text { físico }\end{array}$ & $\begin{array}{c}\text { Desempeño } \\
\text { emocional }\end{array}$ & $\begin{array}{l}\text { Dolor } \\
\text { físico }\end{array}$ & $\begin{array}{c}\text { Salud } \\
\text { general }\end{array}$ \\
\hline $\begin{array}{l}\text { Referencia } \\
\text { versión } \\
\text { española } \\
\text { Edad: } \\
\mathbf{7 5 - 7 9} \text { años }\end{array}$ & 58,3 & 72,9 & 55,4 & 63,1 & $\begin{array}{c}\text { Pendiente } \\
\text { de } \\
\text { desarrollo }\end{array}$ & $\begin{array}{c}\text { Pendiente } \\
\text { de } \\
\text { desarrollo }\end{array}$ & 61,7 & 53,1 \\
\hline $\begin{array}{l}\text { San Ramón, } \\
2010 \\
\text { Recreación } \\
\text { física Pretest } \\
\text { Edad: 50-81 } \\
\text { años }\end{array}$ & 55 & 71,3 & 62,41 & 72,15 & 54,4 & 55,55 & 61,11 & 56,67 \\
\hline $\begin{array}{l}\text { San Ramón, } \\
2010 \\
\text { Recreación } \\
\text { física Postest } \\
\text { Edad: } 50-81 \\
\text { años }\end{array}$ & 74,37 & 94,44 & 85,37 & 91,11 & 90,74 & 91,36 & 85,37 & 77,41 \\
\hline
\end{tabular}

Fuente: Zúniga, Carrillo-Jiménez, Fos, Gandek, y Medina-Moreno, (1999); Sánchez Montero, (2008); Madrigal Jiménez, (2010).

\section{Discusión}

Se ha observado que en personas adultas mayores la actividad física contribuye a prevenir el riesgo de caídas, fracturas y pérdida ósea, a causa del fortalecimiento de los huesos y músculos y la mejoría de la agilidad y la flexibilidad (ACSM, 2007; Cress, y otros, 2005). Se da una disminución de riesgo cardiovascular (CDC, 2010) y también se mejora el bienestar psicológico y permite conservar las habilidades para una vida independiente y autónoma (Stathi, Fox, y McKenna, 2002), todas consecuencias efectivas para una mejor calidad de vida.

La OMS (1998), en su Programa sobre Envejecimiento y Salud, menciona que la independencia es importante pero también lo es, dadas las estructuras sociales humanas, la interdependencia. Señala que para el "envejecimiento saludable" quizás el mejor objetivo que se puede fijar es el de cuidar de uno mismo y de los demás (OMS, 1998). Parte importante de esto es salvaguardar la capacidad funcional y la salud mediante la participación en actividades recreativas dirigidas por profesionales en el área.

En cada una de las dimensiones que evalúa el SF-36, excepto en las de Desempeño físico y emocional, los tres grupos de estudio quedaron arriba del promedio de los valores de referencia españoles (Tabla 6). Los resultados de Madrigal Jiménez (2010) comprueban cambios percibidos estadísticamente significativos en los ocho factores que componen el cuestionario sobre calidad de vida SF-36 luego de concluido el tratamiento (Programa recreativo); estos cambios produjeron en las participantes un estado positivo de bienestar emocional, físico, social y de la salud en general que pudo mejorar la percepción de su calidad de vida. Los promedios del postest reportados son similares a los de los tres grupos de este estudio, un comportamiento muy similar especialmente con el grupo de Baile popular. Según concluye Madrigal, el programa de recreación física grupal mejoró significativamente la percepción de calidad de vida de las participantes, 
lo cual es un estímulo importante para que adopten la recreación como estilo de vida. En el estudio realizado en México (Zúñiga y et ál., 1999) ambos grupos evaluados obtuvieron promedios más bajos en la mayoría de las dimensiones que los tres grupos de este estudio, teniendo un promedio de edad mayor que la población evaluada en México.

Las limitaciones del estudio incluyen el hecho de que para ciertos ítems del SF-36 algunas de las participantes del estudio escribieron justificaciones del porqué de su dolor, del porqué su estado emocional no era el que mantenía la mayoría del tiempo. Por ejemplo, en el ítem 28 una persona escribió: "enviudé hace 2 semanas y entonces sigo triste".

La meta de mejorar la calidad de vida junto con la prevención de estilos de vida no saludables ha cobrado una mayor importancia en la promoción de la salud. Esto es especialmente importante en relación con la satisfacción de las necesidades de las personas de edad avanzada, los enfermos crónicos, los enfermos terminales y las poblaciones discapacitadas (Nutbeam, 1997).

No existen estándares de normalización o percentiles del Test de Raven que clasifiquen y califiquen a una persona que salga bien o mal en este test; sin embargo, al realizarse los cálculos de puntajes $\mathrm{Z}$, se logró evidenciar que los resultados obtenidos por los sujetos de este estudio se ubican en el rango normal de la curva, lo que indica que no fueron puntajes sobresalientes pero tampoco deficientes. Esto indica que las personas evaluadas en este estudio conservan una capacidad cognitiva (habilidades perceptivas, de observación y de razonamiento analógico) buenas o ubicadas dentro del promedio de lo normal.

\section{Conclusiones}

De acuerdo con los objetivos planteados y los resultados de esta investigación se llegó a las siguientes conclusiones:
1. Queda descrita una percepción positiva por parte de los tres grupos de estudio con respecto a su calidad de vida. Los promedios obtenidos en los tres grupos evaluados quedaron arriba del promedio de los valores de referencia españoles.

2. Solamente se encontró diferencia significativa en una de las ocho dimensiones del Cuestionario de Salud SF-36, salud general, entre los grupos de estudio. Esta dimensión es una valoración personal del estado de salud, que incluye la situación actual y las perspectivas futuras y la resistencia a enfermar.

3. No hubo ninguna diferencia significativa con respecto a la capacidad cognitiva en los diferentes grupos de la investigación. Teniendo en cuenta el valor máximo que se podía obtener en esta prueba, los promedios estuvieron dentro del rango normal del área bajo la curva (entre $0 \mathrm{y}+1 \mathrm{DS}$ ).

4. De esta forma se pudo concluir que la capacidad de cognición y percepción de la calidad de vida positiva no dependió del tipo de actividad en la que participó el grupo de investigación.

\section{Recomendaciones}

La expansión y progreso de los programas recreativos que ofrecen las organizaciones e instituciones en el país debe ser un proceso constante y continuo para alcanzar un envejecimiento que dignifique a la población adulta mayor y a la vez debe estar estructurado para obtener beneficios que satisfagan las necesidades de salud física y psicológica de esta población. Se debe tomar en cuenta las recomendaciones publicadas por instituciones que constantemente realizan investigaciones sobre geriatría y gerontología y brindan evidencia científica sobre los mejores procedimientos y métodos al trabajar con este grupo etario. Por ejemplo, las recomendaciones básicas del Colegio Americano de Medicina Deportiva (ACSM) y de la Asociación Americana del Corazón (AHA) en adultos mayores a 65 años o menores con condiciones clínicas crónicas son realizar 30 minutos al día, 5 días a la semana de ejercicio aeróbico de moderada intensidad o 20 minutos por día, 3 días a la semana de ejercicio aeróbico de 
intensidad vigorosa (ACSM, 2007). Para seguir estas recomendaciones, el profesor y/o profesional en salud debe tener claro qué se entiende por intensidad moderada e intensidad vigorosa para constantemente evaluar el esfuerzo durante sus clases.

Es conveniente, si no se tiene de forma impresa y al alcance de la vista de todos los y las participantes la escala de esfuerzo percibido de Borg (la escala de Borg contempla 15 estadios, $6=$ no se siente nada de esfuerzo y $20=$ esfuerzo máximo), utilizar una escala más práctica de 1 a 10, donde 1 es el mínimo esfuerzo y 10 es un trabajo aeróbico fuerte; una intensidad moderada estaría entre 5 y 6 y una intensidad vigorosa entre 7 y 8 . La CDC Center for Desease Control and Prevention 2010 expone criterios muy prácticos para determinar la intensidad a la que se está trabajando, por ejemplo menciona: "si una persona está trabajando a una intensidad moderada sentirá las pulsaciones de su corazón más rápidas, será capaz de hablar pero no de cantar una canción; si está trabajando a una intensidad vigorosa los latidos del corazón incrementarán un poco más su ritmo y no será capaz de decir más que una pocas palabras sin detenerse para recuperar el aliento" (Traducción propia).

Si se trabaja con sesiones que superan la hora de actividad física, como era el caso del grupo de bailes folclóricos y el de actividades físico-recreativas, debe haber una evaluación constante del estado de salud de las personas adultas mayores participantes. Esta evaluación puede realizarse mediante una escala de esfuerzo percibido como se explicó anteriormente, monitoreo de frecuencia cardíaca mediante dispositivo electrónico, toma del pulso sobre la arteria radial con el dedo índice y corazón o por medio de la presión arterial. Preferiblemente el uso de dispositivo electr ónico es mucho más práctico, menos complicado y más preciso para evaluar el esfuerzo realizado siempre y cuando se utilice según las indicaciones de cada fabricante. Además se debe insistir constantemente en la hidratación durante y después de la sesión de actividad física.

El objetivo de las actividades que se planeen nunca debe estar orientado hacia la competencia, sino al bienestar físico, la autoconfianza e independencia de sus actividades motoras diarias. La variabilidad de las actividades es importante para mantener la motivación de esta población de seguir asistiendo a las clases.

Propiciar actividades que involucren contacto social entre los miembros del grupo, promover ejercicios en que la persona adulta mayor no se encuentre aislada, sino más bien se sienta parte del grupo, contribuye a generar seguridad y mayor disfrute a la hora de realizar la clase. En todos los programas en que se trabaje con esta población, es importante brindar espacio y tiempo para que la persona adulta mayor produzca y se sienta parte de la construcción de la clase. Un(a) educador(a) no debe dejar nunca de escuchar a esos alumnos(as) que están deseosos de aprender y experimentar en cada clase actividades que ni se imaginaban capaces de realizar, al saber escucharlos podrán también compartir su conocimiento con el educador o educadora y brindar así una experiencia educativa realmente enriquecedora.

Es necesario seguir realizando más estudios gerontológicos en el país que evalúen la salud de la persona adulta mayor de manera integral y así incentivar la creación de más programas de actividad física y recreación que contribuyan a una buena calidad de vida en esta población. Cada vez son más las personas adultas mayores que se encuentran motivadas a incluir actividades recreativas dentro de su estilo de vida, pero muchas veces no tienen acceso por motivos económicos y/o geográficos; por lo tanto, la misión inclusiva dentro de sus programas de recreación física de instituciones como la Caja Costarricense de Seguro Social, Instituto Costarricense del Deporte y la Recreación, Consejo Nacional de la Persona Adulta Mayor, Asociación Gerontológica Costarricense 
y otros programas institucionales (comités de deportes, universidades, hospitales, empresas privadas, entre otros) es trascendental para mejorar la calidad de vida de las personas de cualquier estrato social residente en cualquier lugar del país.

Los programas recreativos profesionales de ejercicio y/o actividad física para personas adultas mayores deben orientarse a combatir ese círculo vicioso de la inactividad física en que caen por lo general las personas a medida que aumenta la edad (al aumentar la edad, se reduce la actividad física, crece el número de enfermedades crónicas que pueden ser prevenidas o reducidas con la práctica de actividad física) y deben ir encaminados a maximizar la salud emocional, incrementar la movilidad y la inclusión dentro de la sociedad costarricense.

\section{Referencias bibliográficas}

ACSM (2007). Physical Activity and Public Health in Older Adults: Recommendation from the American College of Sports Medicine and the American Heart Association. Medicine \& Science in Sports \& Exercise, 39(8), 1435-1445.

Ballestero Umaña, C. (1998). Fundamentos de la recreación (Manuscrito sin publicar). Universidad de Costa Rica, San José.

Carazo Vargas, P. (2004). Metaanálisis sobre el efecto del ejercicio en el funcionamiento cognitivo en adultos mayores (Tesis de maestría sin publicar). Universidad de Costa Rica, San José.

CDC. (2010). Physical activity is assential to healthy aging. Center for Desease Control and Prevention. Recuperado de: http://www.cdc.gov/physicalactivity/everyone/guidelines/olderadults. html\#Aerobic

Cress, M., Buchner, D., Prohaska, T., Rimmer, J., Brown, M., Macera, C., y otros. (2005). Best Practices for Physical Activity Programs and Behavior Counseling in Older Adult Populations. Journal of Aging and Physical Activity, 13(1), 6-74.

Díaz, V., Díaz, I., Acuña, C., Donoso, A., y Nowogrodsky, D. (2002). Evaluación de un programa de actividad física en adultos mayores. Revista Española de Geriatría y Gerontología, 37(2), 87-92.

Fernández, R. (2005). Teorías sobre el envejecimiento etapas de la edad. Revista digital Investigación y Educación, 3(20), 1-8.

Gracia, M. y Marcó, M. (2000). Efectos psicológicos de la actividad física en personas mayores. Psicothema, 12(2), 285-292.

Grupo WHOQOL. (1996). Evaluación de la calidad de vida. ¿Por qué calidad de vida? Ginebra: Foro Mundial de la Salud, OMS.

Madrigal Jiménez, J. (2010). Percepción subjetiva de cambios en la calidad de vida en mujeres entre los $50 y$ 81 años de edad, residentes de San Ramón, durante su participación en un programa de recreación física grupal (Tesis de maestría sin publicar). Universidad de Costa Rica, San José. Martin, M., Powell, P , Peel, C , Zhu, S , y Allman, R. (2006). Leisure-time physical activity and health-care utilization in older adults. Journal of Aging and Physical Activity, 14(4). 392-410.

Medical Outcomes Trust. (2000). Manual de puntuación de la versión española del Cuestionario de Salud SF-36. Institut Municipal d'Investigació Médica, Barcelona. Recuperado de http://iryss.imim.es/iryss/PDFs/ Manual-Puntuaci\% C 3\% B 3 n SF-36v1.pdf

Nimrod, G. (2007). Retirees leisure: Activities, benefits, and their contribution to life satisfaction. Leisure Studies, 26(1), 65-80.

Nutbeam, D. (1997). Glosario de promoción de la salud del centro colaborador de 
promoción de la salud. Universidad de Sydney, Australia: Confeccionado en nombre de la Organización Mundial de la Salud. Recuperado de http:// www.bvs.org.ar/pdf/glosario_sp.pdf

OMS, Organización Mundial de la Salud. (1998). Envejecimiento Saludable: El envejecimiento y la actividad física en la vida diaria. Programa sobre Envejecimiento y Salud. Ginebra: Organización Mundial de la salud.

Organización Mundial de la Salud. (1986). Concepto de salud. Recuperado de: http://www.who.int/topics/mental_ health/es/

Pont Geis, P. (2007). Tercera edad, actividad física y salud. Teoría y práctica ( $3^{\mathrm{a}}$ ed.). Barcelona: Paidotribo.

Puga, D., Rosero, L., Glaser, K. y Castro, T. (2007). Red social y salud del adulto mayor en perspectiva comparada: Costa Rica, España e Inglaterra. Población y Salud en Mesoamérica, 5(1), 1-21.

Salazar , C. (2007). Recreación. San José, Costa Rica: Editorial UCR.

Salinas, J., Bello, M., Flores, A., Carbullanca, L. y Torres, M. (2005). Actividad física integral con adultos y adultos mayores en Chile: Resultados de un programa piloto. Revista Chilena de Nutrición, 32(3), 215-224.

Sánchez Montero, A. (2008). Efecto de un programa de educación y ejercicio físico en la capacidad funcional $e$ incidencia en el costo de atención en salud en un grupo de personas mayores de 60 años del área de Palmares (Tesis de maestría sin publicar). Universidad Nacional, San José, Costa Rica.

Stathi, A., Fox, K., y McKenna, J. (2002). Physical activity and dimensions of subjective well-being in older adults. Journal of Aging and Physical Activity, 10(1), 76-92.

Wilmore, J. y Costill, D. (2001). Fisiología del esfuerzo y del deporte ( $2^{\mathrm{a}}$ ed.). Barcelona: Paidotribo.

Zúniga, M., Carrillo-Jiménez, G., Fos, P., Gandek, B., y Medina-Moreno, M. (1999). Evaluación del estado de salud con la encuesta SF-36: Resultados preliminares en México. Salud Pública de México, 41(2), 110-118. 